\title{
Development of Automated Warehouse Management System
}

\author{
Deng Mingxing ${ }^{1}$, Mao Jian ${ }^{1}$, Gan Xingwen ${ }^{1}$ \\ ${ }^{1}$ School of mechanical and automotive engineering, Shanghai University of Engineering Science, Shanghai201620, China
}

\begin{abstract}
With the rapid development of the logistics industry, high-speed, high-efficiency and accurate delivery have become the new pursuit goals of the logistics industry, and intelligent logistics has been favored by enterprises. Aiming at the current situation that the offline products must be manually moved, sorted and discharged, the scheme of fully automated three-dimensional warehouse is proposed. Through the design of the whole three-dimensional warehouse, the whole process of the offline products can be realized by AGV, AGV management system, warehouse management system and roller. Lines, palletizers, etc. are completed. As the core part of the whole solution, the warehouse management system plays a vital role in achieving point-to-point tracking of offline products, and can perform regular statistical analysis on the inbound and outbound products to achieve digital management, improve the competitiveness of enterprises and bring considerable economic benefits.
\end{abstract}

\section{INTRODUCTION}

With the promotion of national strategic favorable policies such as "Internet + " and "Made in China 2025" and the increasing demand for informationization, efficient production and intensive warehousing and distribution, the automated warehousing system has been used more and more widely. Its fields of research are also expanding. [1] In recent years, China's express delivery industry has mushroomed, and more and more exports to foreign countries have greatly boosted China's economic growth. As a result, we are also facing new challenges.

In order to meet customers' requirements for product supply efficiency and packaging, as a traditional way, artificial palletizing and film can not meet the actual needs, and can not keep up with the pace of the times. The new technology is applied to realize automatic management of products. In recent years, automated three-dimensional warehouse has become the first choice of major enterprises. This solution is adopted to improve the management efficiency of enterprise warehouses, realize unmanned warehouses, and save a lot of money, which make the enterprise more competitive and vital in the market.

The automated three-dimensional warehouse can also be called an unmanned warehouse. The whole process is carried out by the AGV or the stacker to carry out the inbound and outbound operations, which can reduce the cost of many traditional manual handling of goods. Automated warehouses have the characteristics of high degree of automation, energy saving, safety, intensive storage, high efficiency, and unattended [1]. As the core component of the automated warehouse, the WMS warehouse management system can optimize the distribution of goods entering and leaving the warehouse and track in real time. The warehouse status of the warehouse is also updated in real time as the goods enter and exit, realizing the automatic entry and exit of the goods and the record library. In addition, the WMS warehouse management system can provide warehouse managers with a visual location display, which allows warehouse managers to easily understand the location information and manage the warehouse accordingly.

In view of the many disadvantages of the current offline product storage methods, the automated three-dimensional warehouse can solve these problems well, and finally achieve high efficiency, high quality and digital management of the products.

\section{Demand and site layout}

\subsection{Demand Analysis}

In order to reduce personnel, improve the efficiency of goods outbound, and realize digital management, the company proposes an automated warehouse. It generally includes the following steps: 1 . The offline product is sent to the storage drum line through the latent AGV; 2. The product is sealed and unsealed through the roller line, and then flows into the corresponding crossing; 3 . The fork type AGV will send the crossing goods to the corresponding location allocated by WMS; 4, WMS allocates the outbound task and delivers it to the fork type $\mathrm{AGV}$, and the fork type AGV sends the product to the exit crossing; 5. The products flowing out of the drum line are palletized, covered, and then wrapped around the film and cable tie.

According to the actual research, the daily net storage of the three-dimensional warehouse is about 2,000 cases. 
The goods entering the warehouse need to pass the quality inspection (OQC, Outgoing Quality Control), and the qualified products can be assigned to the warehouse for palletizing. As the core part of the automated warehouse, WMS plays the role of data collection, data statistics, location allocation and information transmission.

\subsection{Site layout}

The automated warehouse is mainly composed of six parts: shelves, containerized unit, conveyor system,

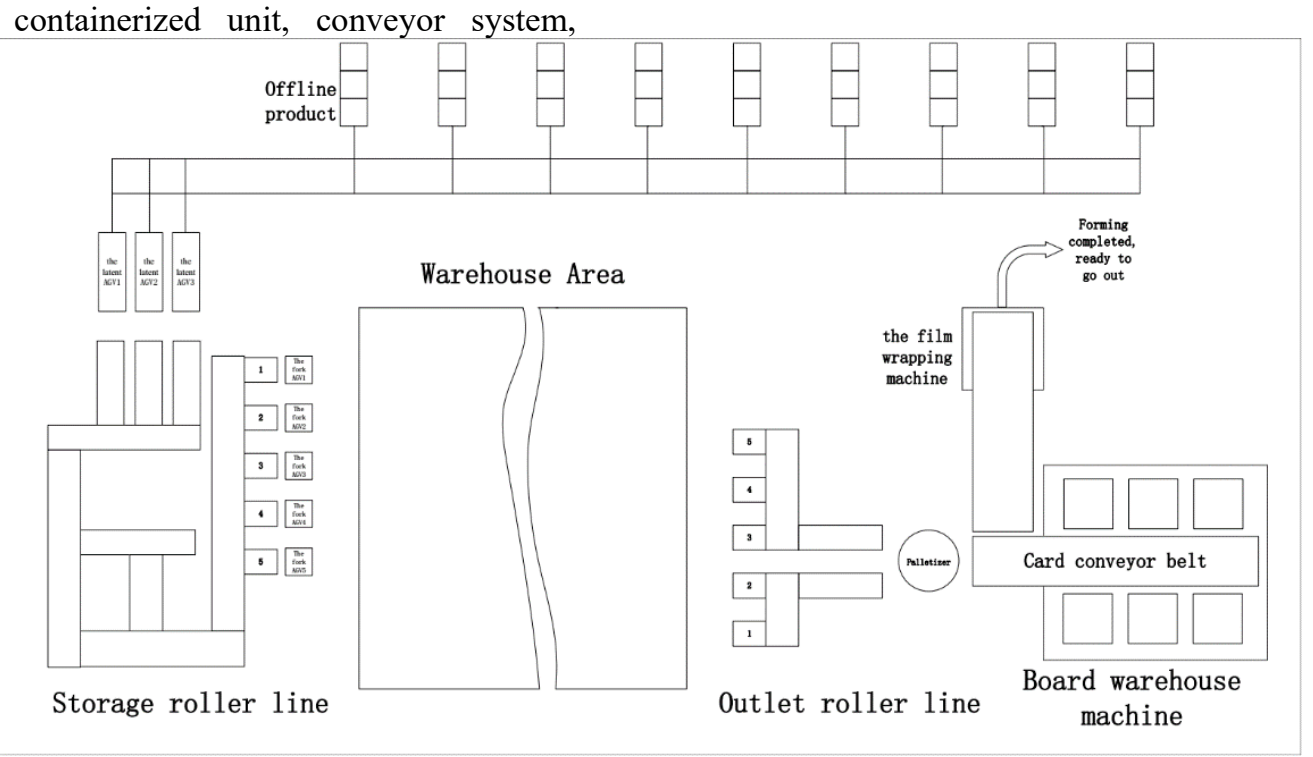

Figure 1. Overall layout

\subsubsection{Warehouse overall layout and location division}

According to the actual demand and on-site venue restrictions, the final number of warehouses determined

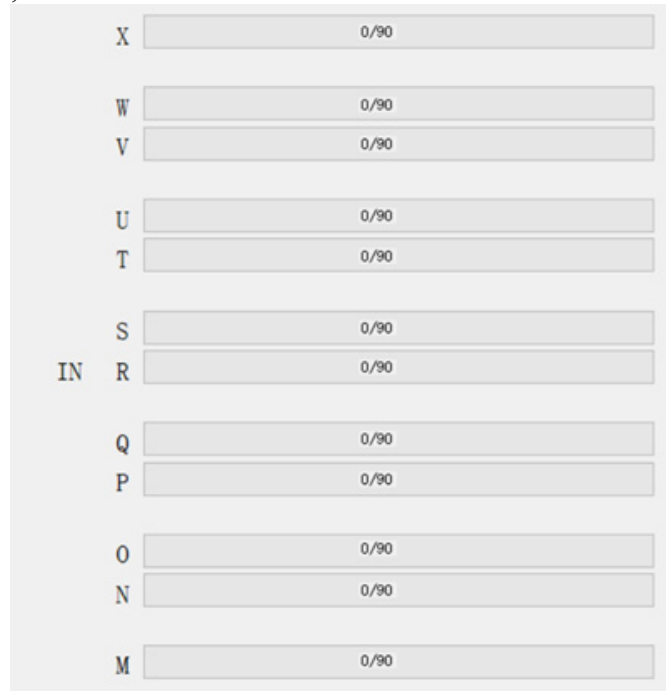

Figure 2. Top view

There are two types of shelf types: Class I includes A $\sim \mathrm{L}$ library area, a total of twelve, each library area has 110 locations; Class II includes $\mathrm{M} \sim \mathrm{X}$ library areas, a total of twelve, each of which has 90 locations.

According to the width of the location and the maximum height of the product, the location categories are divided into four categories: A, B, A1, and B1. automatic control system, and information management system (WMS). [2]

\subsubsection{Overall layout of the site}

The overall layout of the site consists of: the storage roller line, the three-dimensional warehouse, the outbound roller line, the palletizing machine, the film wrapping machine, and the board warehouse machine. The overall layout is shown in Figure 1. by the three-dimensional warehouse is 2,400, divided into 24 areas, which are $\mathrm{A} \sim \mathrm{X}$. The specific layout is shown in Figure 2.

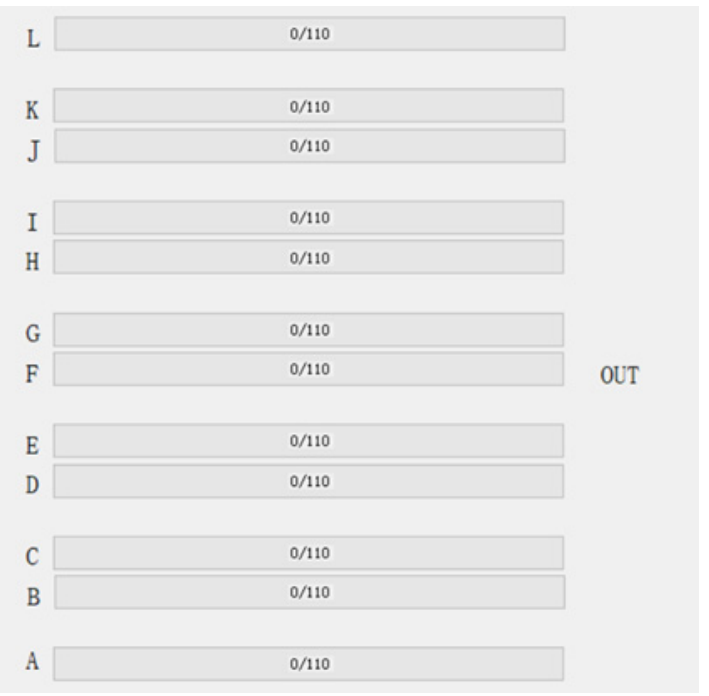

warehouse layout

Class A locations are distributed in the 1-4 layers of the class I library area, with a total of 1056 locations;

Class A1 locations are distributed in the 5th layer of the class I library area, with a total of 264 locations;

Class B locations are distributed in the 1-4 layers of the class II library area, with a total of 864 locations; 
Class B1 locations are distributed in the 5 layer of the class II library area, with a total of 216 locations.
The specific division of the warehouse location categories is shown in Figure 3 and Figure 4.

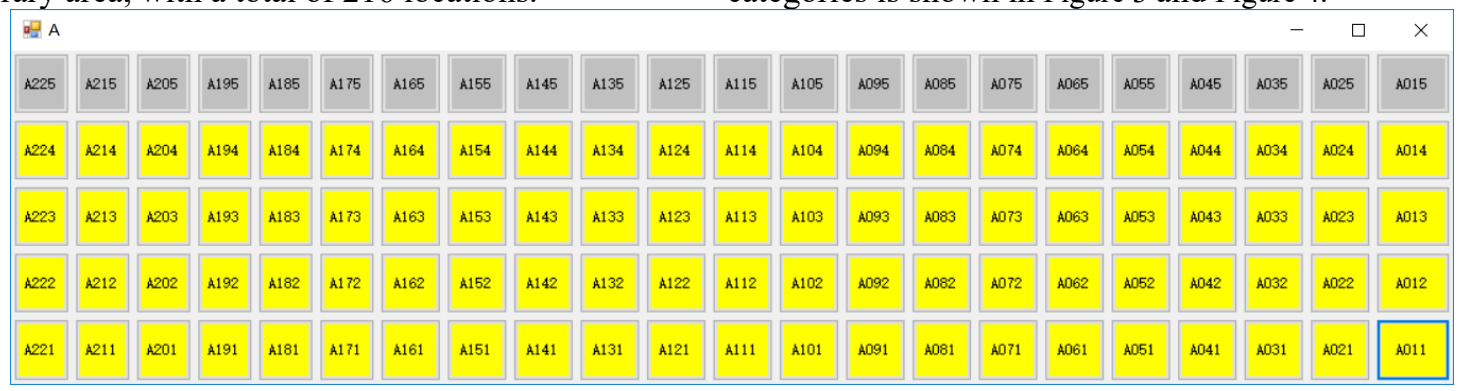

Figure 3. Class I Library Area Location Classification

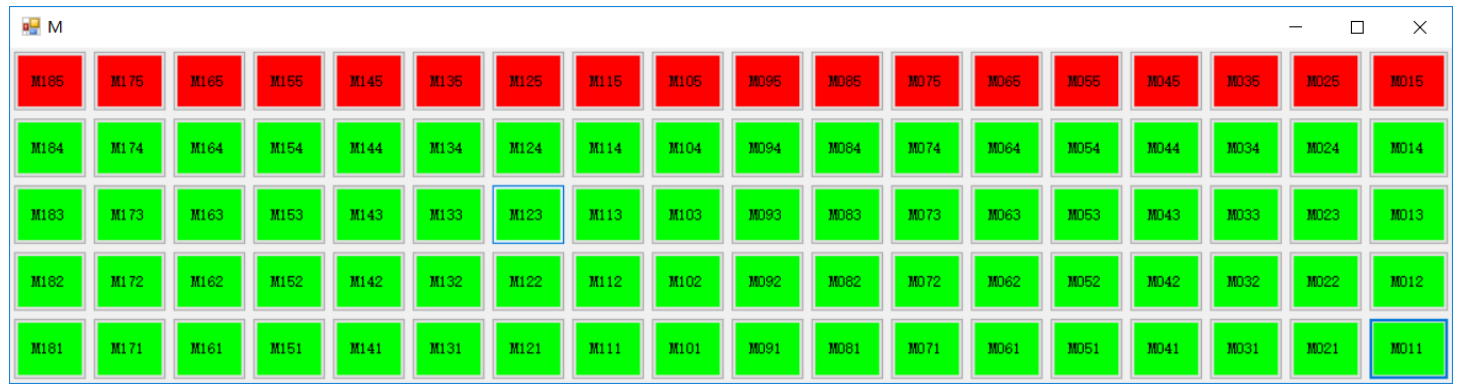

Figure 4. Class II Library Area Location Classification

Description: Among them, yellow is the class A location, grey is the A1 location, green is the B location, and red is the B1 location.

\subsection{System network architecture and communication content}

\subsubsection{WMS system network architecture}

Client-server model Also known as the client-server architecture, C/S architecture, is a network architecture that takes the client (usually a graphical user) The interface program is distinguished from the server. Each instance of the client software can make a request to a server or application server. [3] $\mathrm{C} / \mathrm{S}$ is generally established on a dedicated network. In a small-scale network environment, LANs provide connection and data exchange services through dedicated servers. $\mathrm{C} / \mathrm{S}$ generally targets a relatively fixed user base and has strong control over information security. Generally, the highly confidential information system adopts the $\mathrm{C} / \mathrm{S}$ structure. [4] The field conditions and requirements are basically consistent with the $\mathrm{C} / \mathrm{S}$ characteristics, so the WMS adopts the $\mathrm{C} / \mathrm{S}$ network architecture.

According to the overall plan design of the three-dimensional warehouse, the network framework is built to realize data interaction, and then the normal operation of the three-dimensional warehouse is realized. The network architecture diagram is shown in Figure 5.

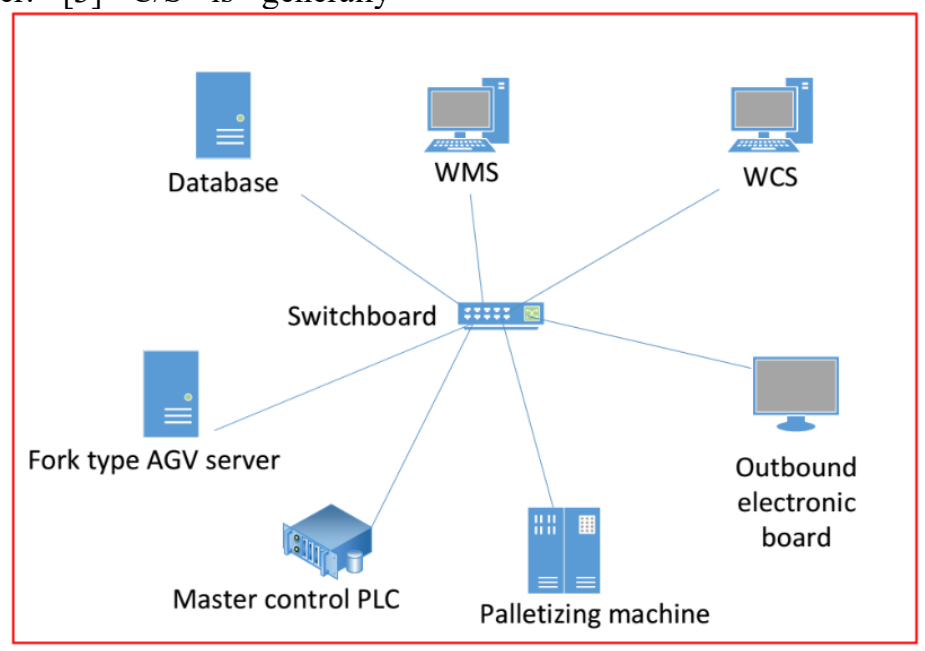

Figure 5. Network architecture diagram

The modules that communicate with WMS are: 1. WCS;

\subsubsection{Communication between WMS and each module}

2. PLC; 3. fork-type AGVS; 4. palletizer 5. database. The communication methods and functions of WMS and each module are as follows. 
WMS communicates with WCS. WCS serves as the data center and provides all the detailed information of the offline products. After the WMS obtains the data, it is used for product location allocation and detailed record recording.

WMS communicates with PLC. PLC obtains scan code information and provides it to WMS, WMS feedback message to roller line

The WMS communicates with the fork AGVS. The WMS sends the task to the AGVS. The AGV performs the task and feedbacks the current status of the task in real time.

The WMS communicates with the palletizer. The WMS sends the palletizing information to the palletizer, and the palletizer performs the correct action.

WMS communicates with the database, and all product data in the warehouse is stored in the database. WMS can be used to change the contents of the database.

\subsection{WMS Main Function}

WMS is written in $\mathrm{C \#}$. The $\mathrm{C \#}$ programming language is a language developed specifically for .NET applications and is a perfect combination with the .NET framework. It differs from some previous programming languages when designing and developing program interfaces. It not only has the characteristics of rapid development of Visual Basic, but also has the powerful functions of $\mathrm{C}++$ language. [5]

WMS is divided into 8 modules, mainly including task status, model management, warehouse management, communication, and business reports.

The task status is used to display the progress of the execution of the currently executing task.

Model management is mainly used to maintain detailed information on the warehousing model.

Warehouse management mainly displays and stores the products in the library.

The communication is mainly used to communicate with each module to realize the correct execution of the WMS function.

The business report is used to perform system statistics on the inbound and outbound product tasks.

Figure 6-8 shows some of the interfaces of WMS:

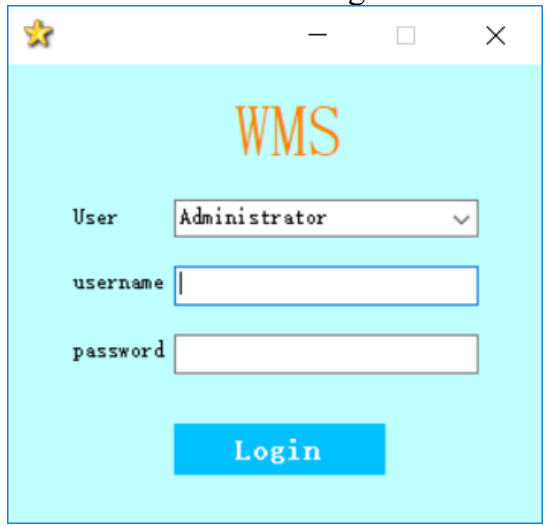

Figure 6. Login window

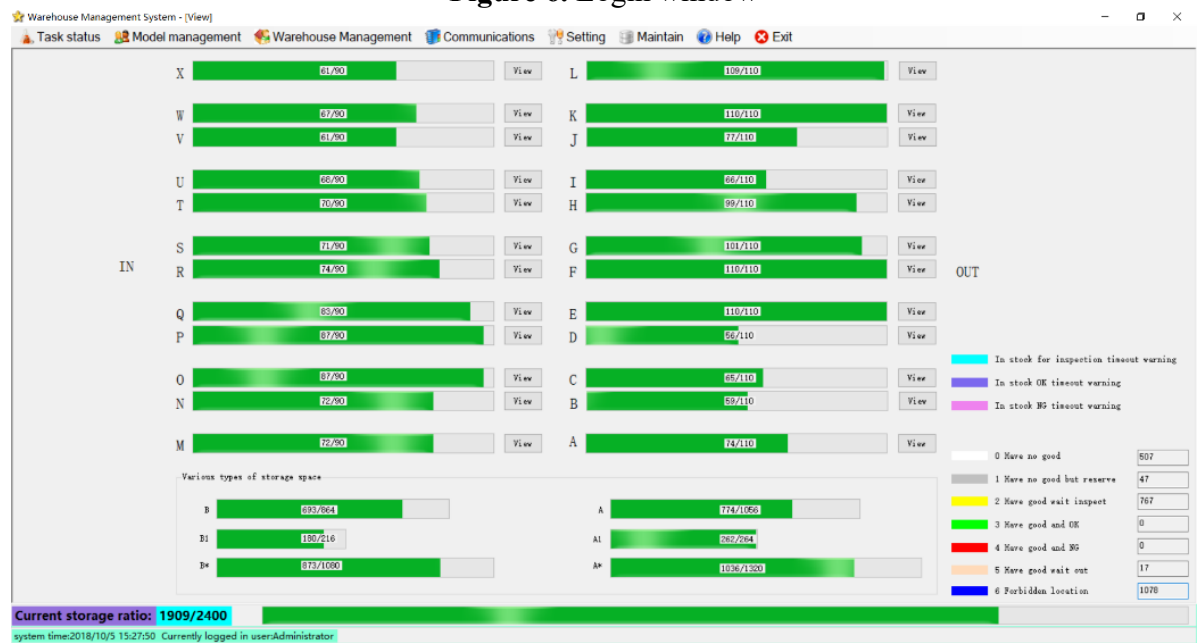

Figure 7. Image Class View Location Status Interface 


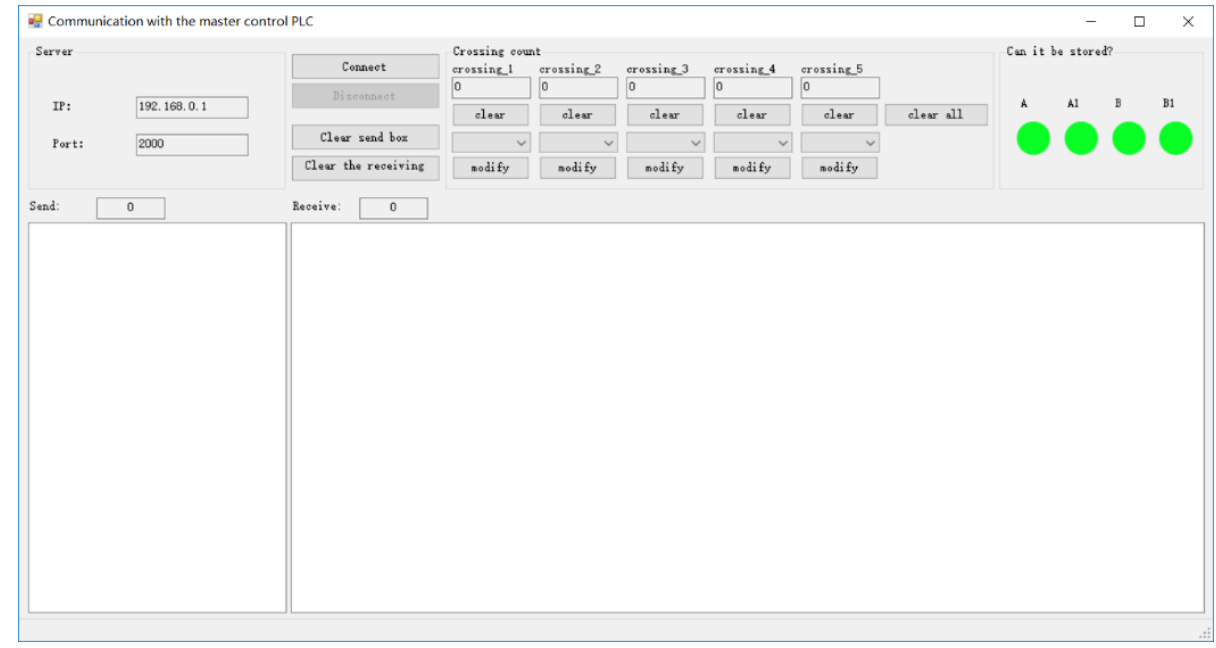

Figure 8. Communication with the master control PLC Interface

\section{Experiment analysis}

Experimental site test process:

Inbound section:

1. The inbound product flows in through the roller line, and the read information is sent to the WMS through the first scanner, and the WMS correctly decodes and sends the sealing information to the roller line console to realize the process of "sealing the product";

2. After the product passes through the second scanner, the read information is sent to the WMS, and the WMS analyzes which type of the product is based on the read information, assigns a corresponding location to generate an AGV inbound task, and At the same time, feedback to the roller line console crossing information, the roller line correctly transports the dispensed product to the corresponding storage crossing;

3 . When the product arrives at the designated inbound crossing, the WMS will deliver the inbound task to the $\mathrm{AGV}$ system, and the AGV will transfer the product to the designated location of the task.

Outbound section:

1. WMS delivers the outbound task to the AGV system according to the quality status and quantity of the library model, and sends the palletizing information, card information, and film information to the palletizer, Card board machine, film machine, to achieve synchronous operation.

2. When the outbound product arrives at the roller line, it needs to go through the scanner. The scanner scans the read information back to the WMS system. The WMS confirms whether it is the current product, and the normal outflow is palletizing. If it is not abnormal, it will flow out, which prevents other products from being mistaken.

After the actual test on site, the above process can be carried out completely without errors, and the reliability of the system is verified. Through the experimental results, it can be concluded that WMS can cooperate with other software and hardware to complete the automatic entry and exit of the product, without manual handling and palletizing, which greatly improves the efficiency of product warehousing.

\section{Conclusion}

As the core of the whole automated warehouse, WMS warehouse management system plays a vital role. As a bridge connecting software and hardware, the WMS warehouse management system realizes the automated storage of off-line products and meets the needs of enterprises for unmanned warehouses and high efficiency. The pursuit of data management has brought competitiveness and vitality to the enterprise, and has created enormous economic value.

This set of automated three-dimensional warehouse solution is different from the previous automated storage solution. The market is mostly a combination of stacking and loading trolleys. This solution is aimed at the existing on-site environment of the enterprise and realizes the automation of the product in the case of minimum on-site transformation. Warehouse management reduces the cost of renovation for the company. The WMS system has a large research space in the location allocation. When the location allocation is further optimized, the speed of the inbound and outbound storage will be further improved. Of course, the AGV walking path needs to be further optimized to realize the location allocation and path. The close coordination of the plan, otherwise there will be many contradictions. When better optimization is achieved, the warehousing efficiency will be greatly improved and the service life of the warehouse shelves will be improved, which is the direction that can be further studied in the future.

\section{References}

1. Liu Xuejun, Li Chengyou. The automatic storage system keeps growing and ushers in greater opportunities $[\mathrm{J}]$. Logistics Technology and Applications, 2017, 22 (04): 57-59.

2. Gan Zhongping.Automated warehousing system that keeps pace with the times[J].Logistics Technology and Application,2016,21(11):93-95. 
3. https://zh.wikipedia.org/wiki/ $\% \mathrm{E} 4 \% \mathrm{~B} 8 \% \mathrm{BB} \% \mathrm{E} 5 \%$ BE $\% 9 \mathrm{E} \% \mathrm{E} 5 \% \mathrm{BC} \% 8 \mathrm{~F} \% \mathrm{E} 6 \% 9 \mathrm{E} \% \mathrm{~B} 6 \% \mathrm{E} 6 \% \mathrm{~A} 7 \% 8 \mathrm{~B}$ ,Wikipedia

4. https://baike.baidu.com/item/B $\% 2 \mathrm{FS} \% \mathrm{E} 7 \% \mathrm{BB} \% 93$ $\% \mathrm{E} 6 \% 9 \mathrm{E} \% 84 / 4868588$ ?fromtitle $=\mathrm{BS} \&$ fromid $=2629$ $117 \&$ fr=aladdin\#3,Baidu Encyclopedia

5. Xia Minjie, Luo Jing. Visual C\#.NET Foundation and Application Tutorial (2nd Edition). Beijing: Tsinghua University Press, 2017:2 